\title{
New perspective on chronic fatigue syndrome: lessons from developmental neuroscience
}

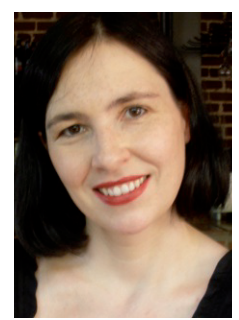

\author{
"Some people believe that the disorder doesn' $\dagger$ \\ even exist and consider CFS a classic \\ manifestation of Freud's concept of hysteria."
}

\section{Christine Heim}

Department of Psychiatry \& Behavioral Sciences, Emory University School of Medicine, 101 Woodruff Circle, WRMB, Suite 4000, Atlanta, GA 30322, USA = Tel.: +1 4047275835 m Fax: +1 4047273233 ncmeim@emory.edu

Hardly ever has a somatic disorder been surrounded with as much controversy as chronic fatigue syndrome (CFS). Some people believe that the disorder does not even exist and consider CFS a classic manifestation of Freud's concept of hysteria, which implies the unconscious simulation of organic disorders, in the context of emotional excitability, high anxiety, and sensory and motor disturbances [1]. Of note, Freud thought that hysteria is brought about by an infantile traumatic experience [1]. At the other end of the spectrum, patient representative groups often vividly advocate CFS as a true medical disease with a sole physiologic cause that, once pinned down, may hopefully be reversible using standard medical techniques in the future.

\section{"Without doubt, CFS is a debilitating condition that is associated \\ with considerable personal suffering and decreased quality of life for affected individuals."}

In an effort to promote CFS as a legitimate medical condition, a nationwide public awareness campaign has recently been launched in the USA, with the aim to dissolve existing myths that surround CFS and educate the public regarding CFS facts [101]. What are some of these facts? First and foremost, the condition is very common with up to $2.5 \%$ of the population suffering from CFS in the USA. CFS affects fourtimes more women than men, and most cases are middle-aged individuals. The average duration of CFS in cases identified from the population is 5-7 years, but less than $20 \%$ have been diagnosed with CFS by a physician. A quarter of affected individuals are unemployed or receive disability benefit and the average household forgoes US $\$ 20,000$ in lost earnings and wages as a result of CFS. The total economic cost of CFS in the USA is an estimated $\$ 9.1$ billion per year. Without doubt, CFS is a debilitating condition that is associated with considerable personal suffering and decreased quality of life for affected individuals $[2,102]$.

Despite the magnitude of the public health problem, the causes of CFS remain unknown and specific targets for prevention and treatment remain elusive. To date, more than 4000 research studies have failed to identify a unanimous cause of CFS and there is evidence that both biological and psychological factors contribute to CFS. Reported biological abnormalities in CFS mostly affect the brain's regulatory outflow systems, namely, the endocrine, autonomic and immune systems. Psychological or behavioral factors contributing to the development or maintenance of CFS include inactivity, avoidance behavior, anxiety sensitivity and stress. High rates of psychiatric comorbidity have been reported for cases with CFS. Behavioral interventions, such as cognitive-behavioral therapy and graded exercise, are among the most effective treatments for CFS, perhaps providing the strongest support for the importance of psychological factors in CFS [3-4].

Patients and clinicians often point out that physical, emotional or chemical stressors trigger the onset of CFS symptoms, an impression also supported by research [5]. As early as in the 1930s, Hans Selye, who first coined the term 'stress', proposed that psychological stress elicits a physiologic response, which he recognized to be adaptive, but that failure of physiological adaptation to stress, or exhaustion of the system, could result in disease [6]. Nowadays, we know that stress exerts multiple effects on the

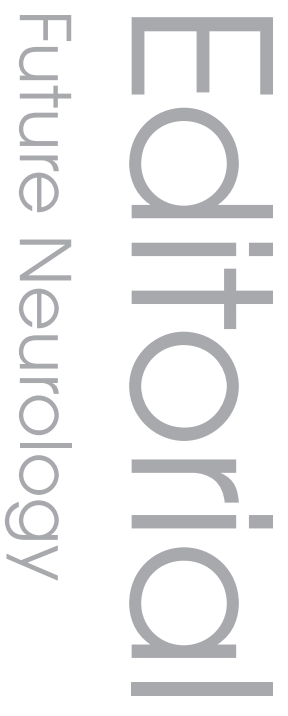

\section{future medican $^{\text {piss }}$}


central nervous, neuroendocrine, autonomic and immune systems that help an organism adapt to challenge. However, much less is known regarding why some individuals fail to adapt, resulting in functional changes that lead to symptoms, such as fatigue, pain, cognitive impairment and sleep disruption, combined with emotional problems. In order to understand CFS, it is of critical importance to understand sources of individual differences in the vulnerability to the pathogenic effects of stress.

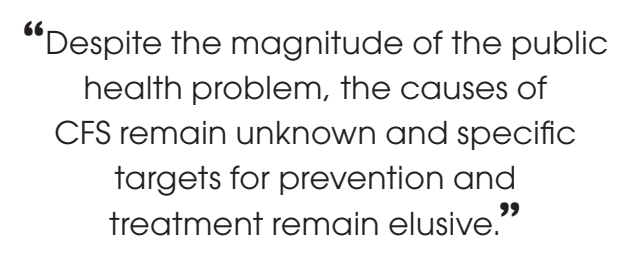

This article discusses results from two recent studies emphasizing the role of childhood trauma in CFS [7-8]. Childhood trauma might be a vulnerability factor that interferes with successful adaptation to stress, thereby conveying a risk to developing CFS. Indeed, there is substantial plasticity of the developing CNS as a function of experience. During such critical periods, certain brain regions are also particularly sensitive to adverse experiences, which may then lead to major, sometimes irreversible, changes [9]. Animal studies, pioneered by the late Seymour Levine [10], provide direct evidence that early adversity permanently programs neural circuits that are involved in regulating the adaptation of physiologic systems and behavioral responses to the environment, by inducing functional, structural and even epigenetic changes in these circuits [11-12]. Among the consequences of early-life stress in animal models and human studies, are many changes that resemble key features of CFS, including decreased basal cortisol secretion, increased immune activation, pain sensitivity, impaired cognition and altered sleep [11-12]. Most recently, it has been ascertained in human post-mortem brain tissue obtained from suicide victims that early adversity associates with epigenetic modulation of a neuron-specific promoter region of the hippocampal glucocorticoid receptor gene, a key regulator of the hypothalamic-pituitary-adrenal (HPA) axis, leading to silencing of the gene by interfering with transcription, which in turn modifies endocrine stress reactivity and behavior [13].

Applying the general principle to CFS, it may be proposed that CFS indeed has an identifiable biological basis, which, albeit unknown, is most likely located at the brain level. At least in some affected individuals, this neurobiological basis may have been shaped by early experience, perhaps in interaction with other factors. As a consequence, the vulnerable brain may inadequately process and/or fail to adapt to emotional, physical or even chemical challenges, thereby producing the often described physiological and behavioral characteristics that form the clinical phenotype of CFS. Clearly, considered from a developmental neuroscience perspective, supported by basic research, it becomes evident that the seemingly disparate points of view regarding the nature of CFS, that is to say psychological versus biological, are not mutually exclusive and that the diverse findings produced by CFS research may well be integrated in a developmental pathway model. Indeed, this perspective offers a new opportunity to reconcile some of the controversy surrounding CFS.

\section{"Among the consequences of early-life stress in animal models and human studies, are many changes that resemble key features of CFS, including decreased basal cortisol secretion, increased immune activation, pain sensitivity, impaired cognition and altered sleep."}

In order to start testing this assumption, it must be demonstrated that; first, early adverse experience is a risk factor for CFS and; second, that this risk factor is associated with a cardinal biological feature of CFS. Towards that end, the CDC (Atlanta, GA, USA) in collaboration with Emory University (Atlanta, GA, USA) recently conducted two population-based studies of CFS cases and controls. The first study employed a sample of 43 clinically confirmed cases with current CFS and 60 controls identified from a general population sample of 56,146 adult residents in Wichita, KS, USA. CFS cases reported significantly higher levels of childhood sexual, physical and emotional abuse, as well as emotional and physical neglect compared with controls. Exposure to childhood trauma was associated with a three- to eight-fold increased risk for CFS, depending on trauma type. Emotional neglect and sexual abuse were the best predictors of CFS. There was a graded relationship between degree of exposure and CFS risk. Childhood trauma was further associated with CFS symptom severity, and with depression, anxiety and post-traumatic stress disorder (PTSD) symptoms. While childhood 
trauma was ascertained as an independent risk factor for CFS, even in the absence of significant psychopathology, the risk of CFS conveyed by childhood trauma increased with the presence of concurrent psychopathology. These results provided support for childhood trauma as an important risk factor of CFS, partly mediated through an altered emotional state [7]. These results, which can be generalized to the population, confirmed and extended similar observations in tertiary clinical samples [14-15].

\section{6}

$$
\begin{aligned}
& \text { maintaining normal cortisol function } \\
& \text { after childhood trauma may protect } \\
& \text { against CFS and other disorders." }
\end{aligned}
$$$$
\text { "...in terms of resilience factors, }
$$

In a second study [8], our group closely replicated the above findings in an independent sample of CFS cases and controls identified from the general population in Georgia, USA. Results of this second study confirmed that emotional maltreatment, and sexual abuse in particular, are potent risk factors of CFS. The risk of having CFS increased to ninefold in the presence of concurrent PTSD symptoms. We then tested whether childhood trauma is associated with a cardinal biological feature of CFS in this sample. One of the hallmark features of CFS is dysfunction of the HPA axis, characterized by lower-than-normal cortisol secretion. Relative hypocortisolism has been observed as a correlate of early-life stress in nonhuman primates and humans, and is also a prominent feature of PTSD. In our study, the CFS group, as a whole, had flattened cortisol levels in response to awakening, in accordance with the general CFS literature. Only when stratifying groups by childhood trauma, it emerged that decreased cortisol secretion in CFS, in fact, was associated with childhood trauma, whereas CFS cases with no childhood trauma, exhibited normal cortisol levels. These results suggested that relative hypocortisolism in CFS, frequently described in the literature, may reflect a biological marker of risk of developing CFS, secondary to childhood trauma, rather than reflecting a correlate of the disorder itself. Similar associations appear to exist with other biological changes in CFS - for example, altered autonomic function.

Although unequivocal evidence for the importance of developmental factors in CFS must come from longitudinal studies, the current data provide important cues regarding potential disease mechanisms and the nature of comorbidity, resilience factors, illness subtypes and intervention strategies related to CFS. Regarding mechanisms, a reduced effect of cortisol as a potential consequence of childhood trauma, may be directly and causally linked to several of the key features of CFS; cortisol secreted during stress mobilizes metabolic resources, by inducing gluconeogenesis, mobilizing free fatty acids and reducing the use of amino acids for protein synthesis. Thus, reduced cortisol effects may decrease the organism's energy resources during stress, leading to exhaustion and fatigue. Cortisol further exerts regulatory effects on the immune system and helps reduce inflammatory responses to challenge. A lack of these cortisol functions may induce an inflammation-like state, promoting fatigue, pain and cognitive problems. Lastly, cortisol has inhibitory effects on stress and emotion systems in the brain. Reduced cortisol effects may thus promote sensitization to stress and anxiety. The combined effects of relative cortisol deficiency might thus promote the symptom complex of CFS and might also explain comorbidity with emotional disorders, based on overlapping pathways [16-17].

“...adopting a developmental neuroscience perspective has significant potential to advance our understanding of CFS. Insights from this line of research may help to overcome the prevailing rejection of the idea that psychological factors may play a role in CFS."

Consequently, in terms of resilience factors, maintaining normal cortisol function after childhood trauma may protect against CFS and other disorders. To emphasize this point, in our study, the average risk of CFS associated with any childhood trauma exposure was increased sixfold [8]. When computing risk ratios depending on childhood trauma exposure and cortisol status, we found that the risk for CFS increased by tenfold for persons who had both a history of childhood trauma and low cortisol secretion. For persons, who had childhood trauma but maintainance of normal cortisol levels, the risk of having CFS ameliorated and was increased approximately threefold [Heim C, Emory University, usa; Maloney M \& Reeves W, CDC, USA, Unpublished Observations]. Thus, maintaining normal cortisol function appears to be a resilience factor that, at least partially, protects against CFS. As a consequence, interventions that normalize the HPA axis and restore cortisol funtion may 
be effective in the prevention or treatment of CFS in children or adults who have experienced early trauma.

\section{"...CFS is clearly a heterogeneous} disorder. There appear to be subgroups of CFS as a function of childhood trauma that are biologically distinguishable."

A final implication of the above results is that CFS is clearly a heterogeneous disorder. There appears to be subgroups of CFS as a function of childhood trauma that are biologically distinguishable. Approximately $60 \%$ of our sample reported at least one form of childhood adversity, while $40 \%$ did not. Changes in neuroendocrine function were limited to the subgroup of CFS cases with childhood trauma histories. The other $40 \%$ had normal cortisol levels, but still suffered from CFS. Perhaps, in analogy to historic approaches to depression classification, the latter group suffers from a more 'endogenous' form of CFS that did not develop in reaction to an environmental event and, therefore, is not associated with altered stress hormone secretion. In fact, there might be even further subgroups not captured by our study design. Thus, an important area for future research is to better define subgroups of CFS and scrutinize diverse mechanisms that may lead to the same clinical picture.

The recognition that there are different subgroups of CFS with different pathophysiologic pathways also helps explain inconsistent findings regarding mechanisms and treatment efficacy in the field. For example, disregarding childhood trauma histories alone likely has significantly confounded previous research on neuroendocrine function in CFS, as demonstrated above. Defining CFS subgroups might also allow for selecting optimal targets for intervention, depending on the individual pathways that led to the disorder. For example, for patients

\section{Bibliography}

1. Freud S: The Aetiology of Hysteria. The Standard Edition of the Complete Psychological Works of Sigmund Freud, Volume III (1893-1899). Early Psycho-Analytic Publications, 187-221 (1896).

2. Reeves WC, Jones JF, Maloney E et al.: Prevalence of chronic fatigue syndrome in metropolitan, urban, and rural Georgia. Popul. Health Metr. 5, 5 (2007).

3. Prins JB, van der Meer JW, Bleijenberg G: Chronic fatigue syndrome. Lancet 367(9507), 346-355 (2006).
4. Afari N, Buchwald D: Chronic fatigue syndrome: a review. Am. J. Psychiatry. 160, 221-236 (2003).

5. Salit IE: Precipitating factors for the chronic fatigue syndrome. J. Psychiatr. Res. 31, 59-65 (1997).

6. Selye $\mathrm{H}$ : The general adaptation syndrome and the diseases of adaptation. Practitioner 163, 393-405 (1949)

7. Heim C, Wagner D, Maloney E et al.: Early adverse experience and risk for chronic fatigue syndrome: results from a population- with chronic depression and irritable bowel syndrome, it has been demonstrated that those with childhood trauma experiences benefitted from different treatments than those without such experiences [18-19].

In conclusion, adopting a developmental neuroscience perspective has significant potential to advance our understanding of CFS. Insights from this line of research may help to overcome the prevailing rejection of the idea that psychological factors may play a role in CFS, at least for a proportion of cases. Perhaps patients and advocacy groups fear to be labeled with the stigma of 'simulating' symptoms, as initially suggested by Freud. However, modern neuroscience clearly demonstrates that experience shapes biology (and vice versa) and, in this way, can create 'real' organic symptoms. Freud's legacy is that he was remarkably correct in recognizing that early-life trauma is a major risk factor for a variety of functional disorders. However, it was Hans Selye who realized that successful biological adaptation to stress is critical to maintain health. The key to CFS might lie in the combination of both points of view, based on indisputable advances from modern developmental neuroscience.

\section{Financial \& competing interests disclosure}

The work discussed in this editorial was supported by the CDC (Atlanta, GA, USA). Christine Heim has received/receives funding or fees from National Institute of Mental Health, National Alliance for Research in Schizophrenia and Depression, Anxiety Disorders Association of America, Eli Lilly, Novartis and CeNeRx. The author has no other relevant affiliations or financial involvement with any organization or entity with a financial interest in or financial conflict with the subject matter or materials discussed in the manuscript. This includes employment, consultancies, honoraria, stock ownership or options, expert testimony, grants or patents received or pending, or royalties.

No writing assistance was utilized in the production of this manuscript.

based study. Arch Gen Psychiatry 63, 1258-1266 (2006).

8. Heim C, Nater UM, Maloney E et al.: Childhood trauma and risk for chronic fatigue syndrome: association with neuroendocrine dysfunction. Arch. Gen. Psychiatry 66, 72-80 (2009).

9. Weiss MJ, Wagner $\mathrm{SH}$ : What explains the negative consequences of adverse childhood experiences on adult health? Insights from cognitive and neuroscience research. Am. J. Prev. Med. 14, 356-360 (1998). 
10. Levine S: Maternal and environmental influences on the adrenocortical response to stress in weanling rats. Science 156(772), 258-260 (1967)

11. Meaney MJ: Maternal care, gene expression, and the transmission of individual differences in stress reactivity across generations. Annu. Rev. Neurosci. 24, 1161-1192 (2001).

12. McEwen BS: Understanding the potency of stressful early life experiences on brain and body function. Metabolism 57(Suppl. 2), S11-S15 (2008)

13. McGowan PO, Sasaki A, D'Alessio AC et al.: Epigenetic regulation of the glucocorticoid receptor in human brain associates with childhood abuse. Nat. Neurosci. 12, 342-348 (2009).

14. Van Houdenhove B, Neerinckx E, Lysens R et al.: Victimization in chronic fatigue syndrome and fibromyalgia in tertiary care: a controlled study on prevalence and characteristics. Psychosomatics 42, 21-28 (2001).
15. Fisher L, Chalder T: Childhood experiences of illness and parenting in adults with chronic fatigue syndrome. J. Psychosom. Res. 54, 439-443 (2003).

16. Heim C, Ehlert U, Hellhammer DH: The potential role of hypocortisolism in the pathophysiology of stress-related bodily disorders. Psychoneuroendocrinology 25, 1-35 (2000).

17. Raison CL, Miller AH: When not enough is too much: the role of insufficient glucocorticoid signaling in the pathophysiology of stress-related disorders. Am. J. Psychiatry 160, 1554-1565 (2003).

18. Nemeroff CB, Heim CM, Thase ME et al.: Differential responses to psychotherapy versus pharmacotherapy in patients with chronic forms of major depression and childhood trauma. Proc. Natl Acad. Sci. USA 100, 14293-14296 (2003).

19. Creed F, Guthrie E, Ratcliffe J et al.: Reported sexual abuse predicts impaired functioning but a good response to psychological treatments in patients with severe irritable bowel syndrome. Psychosom. Med. 67, 490-499 (2005).

\section{Websites}

101. Chronic fatigue syndrome: public awareness campaign. www.cfids.org/sparkcfs/default.asp

102. Centers for Disease Control and Prevention. Chronic fatigue syndrome. www.cdc.gov/cfs/cfsbasicfacts.htm

\section{Affiliation}

- Christine Heim, PhD Associate Professor, Department of Psychiatry \& Behavioral Sciences, Emory University School of Medicine, 101 Woodruff Circle, WRMB, Suite 4000, Atlanta, GA 30322, USA

Tel.: +14047275835

Fax: +1 4047273233

cmheim@emory.edu 